\section{Life after Cardiopulmonary Resuscitation in Patients with Cardiovascular Diseases} ORIGINAL

\section{Abstract}

Background: Evaluating the quality of life in patients surviving cardiac arrest after discharge from hospital.

Methods and Findings: The study included 105 patients survivors of a cardiac arrest admitted in Intensive Care Unit at Institute of Cardiovascular Diseases „Prof. Dr. George I.M. Georgescu”, lasi. Over 5 years patients were interviewed by phone or medical reevaluation, after their verbal consent. We applied the Romanian version of the Short Form 36 questionnaire. Eight scales of SF-36 health survey were scored: physical functioning (PF), role of physical functioning (RP), body pain $(B P)$, general health $(G H)$, vitality $(V T)$, social functioning $(S F)$, role of emotional functioning (RE) and mental health (MH).

The scales scores of PF, RP, RE, VT, MH, SF, BP and GH were as follows: $57.4 ; 22.2 ; 30.28 ; 50.67 ; 54.14 ; 76 ; 71 ; 48.5$. Comparing the dates with the scores available in the literature for the general population we observed good general scores for the physical items while the emotional items are generally low; most of the patients limited their physical activity, mostly due to emotional perception about acute event.

Conclusions: Post resuscitation, the survivors have an acceptable quality of life, justifying the medical and financial efforts.

\section{Introduction}

Nowadays, it is unanimously accepted that a successful resuscitation depends on adequate ventilation and circulation. The main long term objective of both resuscitation and post cardiac arrest care is recovering health quality, and mainly the neurological sta-
Liviu Macovei1,2,

Dan Mihai Alexandrescu2,

Alina Iliescu2,

Ana Tanasa ${ }^{2}$

Larisa Anghel2,

Catalina Arsenescu

Georgescu $^{1,2}$

1 University of Medicine and Pharmacy "Grigore T. Popa", Iasi, Romania.

2 Institute of Cardiovascular Diseases "Prof. Dr. George I.M.Georgescu" Iasi, Romania.

Contact information:

Liviu Macovei.

Address: Intitute of Cardiovascular Diseases "Prof. Dr. George I. M.

Georgescu", Carol I Boulevard no. 50, lasi, 700503, Romania..

झ liviughemacovei@yahoo.com

Keywords

Morbidity; Cardiopulmonary

Resuscitation; Heart Arrest;

Cardiovascular Disease. 
tus. Even if the patient's personal perception on his own health status could be the most appropiate instrument to evaluate a successful resuscitation, the evaluation of quality of life is not a part of actual clinical examination routine [1, 2]. Concerns have been expressed about an unacceptable quality of life, which wouldn't justify the medical efforts and the costs involved. In other words, a current dilemma in the medical world is generated by essential ethical aspects of cardiopulmonary resuscitation: autonomy, beneficence and futility. Are they respected and applied correctly? [3, 4] The question we posed is: what is the real quality of life of the survivors of a cardiac arrest after hospital discharge?

Of the 70 studies available, 46 concluded that the quality of life postresuscitation is mostly good, only 7 having negative conclusions and 17 being neutral, underlining the discrepancy between the good physical health and the poor quality of life due to cognitive and social status alterations. The cognitive impairment make the available questionnaires lack sensitivity and specificity, requiring specialist expertise. $[3,5]$

Quality of life is difficult to assess. The modern concept of "health related quality of life" (HRQoL) derives of the World Health Organization definition of health, "a state of complete physical, mental and social well-being, not merely the absence of disease or infirmity". Quality of life includes three fundamental domains: biological, psychological and social functioning, influenced by a large number of variables, including personal perception. [3] The validated assessment tools for quality of life are either questionnaires (Health Utilities Index 3, Nottingham Health Profile, Everyday Life Questionnaire) and score systems allowing the comparison with representative control lots.

\section{Aim}

The purpose of our paper was to evaluate quality of life in patients surviving a cardiac arrest, the perceptions of the patient and family of the medical care and medical decisions and about the acute event.

\section{Material and Methods}

In order to examine the real quality of life of cardiac arrest survivors after hospital discharge, we used the Short Form Questionnaire (SF-36), a validated health survey, developped and tested by New England Medical Center, adapted from Medical Outcomes Study Questionnaire, highlighting eight health scales: physical functioning (PF), role of physical functioning (RP), body pain (BP), general health $(\mathrm{GH})$, vitality (VT), social functioning (SF), role of emotional functioning (RE) and mental health (MH). [6] SF-36 is useful to evaluate patients with single or multiple pathological conditions and to compare their health to general population health. The Romanian version was developped according to the internationally instructions accepted, under the supervision of New England Medical Center. Items are scored on a 0 to 100 range, a high score defining a favorable health state. Scores represent the percentage of total possible score achieved. [6-8]

105 survivors of a cardiac arrest, admitted to Institute of Cardiovascular Diseases „Prof. George I.M. Georgescu" Iaşi, in the Intensive Care Unit, over 5 years, were interviewed by phone or medical reevaluation after obtaining their verbal consent, right after or during the hospital stay. We applied the Romanian version of the Short Form 36 questionnaire. We used as inclusion criteria the following: age above 18, survivors of a cardiac arrest, the ability and accept of the patient to speak. The results were compared to a representative control lot (taken from the existing international studies). $[3,7,9]$

The study was approved by an institutional review committee and all the subjects gave verbal consent. 


\section{Results}

Most of the patients included in our study had coronary artery disease (82, respectively 78\%), 11 patients $(10 \%)$ were diagnosed with total atrioventricular block, 12 patients (6\%) presented malign ventricular arryhthmia due to hypertrophic obstructive cardiomyopathy, long QT syndrome or pulmonary tromboembolism. 5 patients had an implantable cardioverter-defibrillator.

The general scores obtained for every scale are detailed in Table 1.

Table 1. Life quality postresuscitation scores.

\begin{tabular}{|l|c|c|}
\hline Scale & Medium score & General population score \\
\hline PF & 57.4 & 84.2 \\
\hline RP & 22.2 & 81 \\
\hline RE & 30.28 & 81.3 \\
\hline VT & 50.67 & 60.9 \\
\hline MH & 54.14 & 74.7 \\
\hline SF & 76 & 83.3 \\
\hline BP & 71 & 75.2 \\
\hline GH & 48.5 & 72 \\
\hline
\end{tabular}

On integrating patients responses, we observed significant limitation in physical activity, either due to the natural evolution of the main disease (e.g. severe cardiac failure) or due to emotional perception of the acute event ("I was nearly dead"). The majority of patients limited the type, the level and the time for physical activity, not always symptomlimited but often due to lack of information about the safety of different types of physical activity or exaggerated anxiety (patients or their family). A further difficulty in interpreting the questionnaires was that the patients were asked to compare their present life situation with the quality of life they had prior the cardiac arrest, this leading to the negative responses given. The alternative to their current situation (if no resuscitation had been provided) would be death or severe neurological damage. [3, 9-11] Interesting, the patients included in rehabili- tation programmes (a small percent), most of them educated people, or encouraged to come back to their proffessional activity had better scores for physical and emotional health.

The psychological impact of the near-death experience significantly affects the survivors' perception on their own life. [5] Most of the patients with low emotional scores had no familial or social support. Social isolation, depression, and anxiety cause physical activity limitation and mental disconfort, due to comparing their present situation to the years free of illness. [9, 10]

The majority of patients interviewed completely recovered their neurological function. There were 13 cases with persistent postanoxic neurological deficits, mostly cognitive impairment with memory deficits of different severity, personality changes, emotional lability or parapsychotic disorders. Patients with severe neurological impairment were not included in this study, not being able to communicate.

A factor with major impact on postresuscitation quality of life was the patient's age when the acute event occured. The general score and the emotional scores were superior in patients under 50 years old, most of them being socially reintegrated.

\section{Discussion}

Cardiopulmonary resuscitation is performed most of the time in emergency, before the physician has the time to go through all the steps of a certain diagnosis (physical exam, laboratory findings, imaging studies). In this situation, because of the absence of a certain diagnosis and the correlations with short and long term prognosis after cardiopulmonary resuscitation, the options in front of some ethical problems are limited. Therefore, in order to evaluate ethically this decision, but also to maintain medical conscience, the physician should have an affirmative answer to these 3 questions, evaluated as a test: $[1,12]$ 
- test of impartiality: would he agree to the same action applied to himself, in similar situation?

- test of universality: in the same circumstances, do all the physicians apply the same method?

- test of justification: is there a justification for these actions?

The key principles of ethics are referred to as autonomy, beneficence, non-maleficence, justice, dignity and honesty.

Autonomy is the right of the pacient to refuse or to accept any treatment. As a doctor, we are sometimes asked by people around us - family, friends, colleagues - not to "torture" them and let them die with dignity when they will be in a critical situation, like cardiopulmonary resuscitation,. Although most of the time we consider it "just words", we should take in account the legal and ethical problems that could ensue when the patient's autonomy principle is not well understood or applied. [12]

The right of the pacient to an informed decision should take precedence to the medical decision. Autonomy requires that the patient is informed, competent, free from any pressure. However, it is very difficult to apply this principle in an emergency, such as sudden cardiac arrest, when treatment must be applied in the absence of evidence of patient decision.13,14

In this regard, some countries adopted an advanced directive which clearly provides patient's choice in case of cardiopulmonary resuscitation, or designates a surrogate person to decide. For example, in the United States of America, the legal interruption of a life maintaining therapy can applied only if: [12]

- the surrogate person gives his/her consent;

- 2 physicians conclude that the disease is terminal;

- the patient is in a persistent vegetative state, documented by 2 doctors, one of them is a specialist in evaluating cognitive functions.

Beneficence, balancing risks for the patient's best interest, involves taking the decision between at- tempting, withholding or stopping the resuscitation. Doing no harm (or no further harm) is the principle that applies in obviously futile cases.

A major principle in the ethics of cardiopulmonary resuscitation is the one of qualitative and quantitative uselessness, a principle which concerns the quality and span of life after resuscitation. Otherwise put, it is considered that any intervention is useless if it is not followed by an increase lifetime and quality of life. The major criteria underlining the qualitative uselessness principle are: pre-existing pathology and post-resuscitation status.

Thereby, cardiopulmonary resuscitation should not be performed when chances of survival are null, or when the patient would survive but would lack the possibility of talking. [12, 15]

The principle of uselessness raises more ethical problems: [12]

- discrepancy in the evaluation of quality of life between physicians and survivors or their family;

- the quality of life is differently seen from a legal, cultural and religious point of view by survivors or by their family.

These aspects have created a medical and public debate which tries to answer to some key questions: [12]

- what is the estimated survival rate at which you don't start cardiopulmonary resuscitation?

- increasing survival with 1-2 months in patients with end-stage disease merits cardiopulmonary resuscitation?

- who decides what's futile: the physician, the patient, the family or a committee of experts? Justice implies the concern and duty to equally distribute health resources to patients that need them. $[13,16,17]$

Dignity and honesty are essential principles of ethics. Patient have the right to be well informed transparently, the physician treating them disclosing conflicts of interests. [13, 16, 17] 
Resuscitation efforts are focused on unexpected cardiac arrest that should have been prevented. The decision on commencing the resuscitation should consider the patient's chance to a fair quality of life post-resuscitation. Death is inevitable in $70-98 \%$ of cases. Several studies concluded to an acceptable quality of life post-resuscitation. The clinician must inform the patient and treat the potential problems after a successful resuscitation: anxiety, depression, cognitive difficulties. [18-20]

In all cases, the doctor has to take the decision to start or stop resuscitation attempts, balancing the benefits, the risks and also the costs of the medical system. [21, 22]

Resuscitation is inappropriate in futile cases or against expressed wish of the patient.

This is a difficult point of discussion, due to the deficient legislation, although guidelines on do not resuscitate orders are recently available. The protocols differ for variable legislations, culture or local traditions. [13, 18, 19]

What are futile cases? Futility exists if the resuscitation will bring no benefit in terms of prolonging life of acceptable quality. This is an individual decision and there are still uncovered areas that allow subjective decisions: severe heart failure, severe respiratory compromise, major trauma, neurological deficits. $[13,17,20]$ Age is frequently a factor of decision. The health provider will insist in resuscitating a child, due to emotional factors. However, high age means an association of multiple comorbidities, severely affecting the prognosis. [23-25]

The survivors of a cardiac arrest without neurological impairment have a good quality of life, although not as good as before the acute event, depending not only on physical parameters, but also on psychological markers such as depression and anxiety. Thus, the satisfactory long term prognosis, depending also on the severity of underlying illness, justify medical and financiary efforts. [11]

Although quality of life is rarely assessed as an important part of clinical examination and there aren't specific instruments to evaluate it, it remains one of the most important long term objectives for the patients surviving a cardiac arrest. The pharmacological treatment, and periodical medical examinations are insufficient, therefore we should also focus on adequate psychological, familial, social, or proffessional support, whenever needed. The doctor-patient relationship, informing the patient about his illness and encouraging the rehabilitation programmes remain important items to improve long term outcome. [26]

The literature on the subject does not offer many guidelines about the decision to end life, a complex multi-factorial decision, bringing in discussion individual, international, cultural, religious, socio-economic aspects and opinions. To make matters worse, often these difficult decisions are taken in the emergency room, without prior informing. This is when the principles of ethics become very useful in making the right choice. The 2010 resuscitation Guidelines, emitted by the European Council of Resuscitation, contains a special section for ethics. [13] Mostly, the decision has to be taken in the emergency department, in the absence of previous information of the patient and that's why ethical principles are extremely useful for doctors in establishing their medical behavior. On the other side, they are expected to provide a series of answers to ethical problems that autonomy, benefit or uselessness principle induce in the cardiopulmonary resuscitation. A careful medical analysis of the clinical-prognosis and ethical implications could remove the subjectivism and could complete the actual ethical guidelines in cardiopulmonary resuscitation. Also, we consider that the development of ethical guidelines in cardiopulmonary resuscitation is much needed, for the judgment of sensitive current problems and the existence in hospitals of some interdisciplinary ethical committee. Only in this way, in consensus with civil society, and with "medical art" and compassion, will we be able to take remedial and ethical action of cardiopulmo- 
nary resuscitation; we can prevent social isolation and increase quality of life post-resuscitation.

\section{References}

1. Colqueboun MC, Handley AJ, Evans TR. ABC of Resuscitation 5th ed. BMJ Publishing Group, 2004.

2. Cimpoesu D, Rotariu I, Costache I, Petris A. Decizia "de a nu resuscita" (II). Etică şi lege în resuscitarea cardiopulmonară. Revista Română de Bioetică. 2012; 10(2): 29-41. Romanian

3. Elliott JV, Rodgers LD, Brett JS. Systematic review of quality of life and other patient-centred outcomes after cardiac arrest survival. Resuscitation.2012;82: 247-56.

4. Holm S, Jorgensen EO. Ethical issues in cardiopulmonary resuscitation. Resuscitation.2001; 50(2):135-9.

5. Pearn J. Successful cardiopulmonary resuscitation outcome reviews. Resuscitation. 2004; 47: 311-16.

6. Mc Horney CA, Ware JE, Raczek AE. The MOS 36-item short form health survey (SF-36)II. Psychometric and clinical test of validity in measuring physical and mental health constructs. Medical Care. 1993; 31(3):247-63.

7. Stiell I, Nichol G, Wells G, De Maio V, Nesbitt L, Blackburn J et al. Health-Related Quality of Life Is Better for Cardiac Arrest Survivors Who Received Citizen Cardiopulmonary Resuscitation. Circulation.2003;108: 1939-44. doi:10.1161/01. CIR.0000095028.95929.B0

8. Wachelder EM, Moulaert VT, van Heugten C, Verbuntb JA, Bekkers SC, Wade DT. Life after survival: Long-term daily functioning and quality of life after an out-of-hospital cardiac arrest. Resuscitation. 2009; 80: 517-22. doi:10.1016/j. resuscitation.2009.01.020

9. Lundgren-Nilsson A, Rosen H, Hofgren C, Stibrant Sunnerhagen $K$. The first year after successful cardiac resuscitation: Function, activity, participation and quality of life. Resuscitation. 2005; 66: 285-89. doi: http://dx.doi.org/10.1016/j. resuscitation.2005.04.001

10. Laua B, Kirkpatrick JN, Merchanta RM. Experiences of sudden cardiac arrest survivors regarding prognostication and advance care planning. Resuscitation.2005; 81: 982-86.

11. Saner H, Borner-Rodriguez E, Kummer-Bangerter A, Schuppel $\mathrm{R}$, von Planta M. Quality of life in long-term survivors of out-ofhospital cardiac arrest. Resuscitation. 2002; 53: 7 -13.

12. Cardiopulmonary resuscitation guide in adult. Ethical issues in resuscitation. http://www.cardioportal.ro/files/pdf/ghiduri/ Ghid\%20\%de\%20Resuscitare.pdf. Romanian

13. Lipperta FK, Raffayb V, Georgiouc M, Steend PA, Bossaert L. European Resuscitation Council Guidelines for Resuscitation 2010, Section 10. The ethics of resuscitation and end-of-life decisions. Resuscitation. 2010; 81: 1445-51.
14. Association World Medical. Declaration of Helsinki Ethical principles for medical research involving human subjects adopted by the $18^{\text {th }}$ WMA General Assembly Helsinki, Finland, June 1964 and amended at the $29^{\text {th }}, 35^{\text {th }}, 41^{\text {st }}, 48^{\text {th }}, 52^{\text {nd }}, 5^{\text {th }}$ and 59th WMA Assemblies. Helsinki: World Medical Association, 1964.

15. Ethical Aspects of CPR and ECC. http://circ.ahajournals.org/ content/102/supp|1//-12.full\#sec-19.

16. Horsted TI, Rasmussen LS, Lippert FK, Nielsen SL. Outcome of out-of-hospital cardiac arrest-why do physicians withhold resuscitation attempts? Resuscitation. 2004;63:287-93. doi:10.1016/j.resuscitation.2004.05.005

17. Morrison LJ, Visentin LM, Kiss A, Theriault R, Eby D, Vermeulen $M$ et al. Validation of a rule for termination of resuscitation in out-of-hospital cardiac arrest. N Engl J Med. 2006; 355:478-87.

18. Morrison LJ, Verbeek PR, Zhan C, Kiss A, Allan KS. Validation of a universal prehospital termination of resuscitation clinical prediction rule for advanced and basic life support providers. Resuscitation. 2009; 80:324-28. doi: 10.1016/j. resuscitation.2008.11.014. Epub 2009 Jan 15.

19. Richman PB, Vadeboncoeur TF, Chikani V, Clark L, Bobrow BJ. Independent evaluation of an out-of-hospital termination of resuscitation(TOR) clinical decision rule. Acad Emerg Med. 2008; 15:517-21. doi: 10.1111/j.1553-2712.2008.00110.x.

20. Skrifvars MB, Vayrynen $T$, Kuisma $M$, Castren M, Parr MJ, Silfverstople J et al. Comparison of Helsinki and European Resuscitation Council "do not attempt to resuscitate" guidelines, and a termination of resuscitation clinical prediction rule for out-of-hospital cardiac arrest patients found in asystole or pulseless electrical activity. Resuscitation. 2010; 81:679-84. doi: 10.1016/j.resuscitation.2010.01.033. Epub 2010 Apr 8.

21. Fendler T, Li Y, Spertus JA, Chan PS. Defining the association of do-not-attempt-resuscitate orders with patients' likelihood of survival after in-hospital cardiac arrest. Circulation Cardiovasc Qual Outcomes. 2014; 7 Suppl 1:A3. circoutcomes.ahajournals. org/content/7/Suppl_1/A3.abstract

22. Young TJ, Perman S, Daeschler M, Kolansky D, Gaieski D, Kirkpatrick JN. Ethics of codes: provider perspectives on cardiopulmonary resuscitation duration. Circulation Cardiovasc Qual Outcomes. 2014; 7 Suppl 1: A187.

23. Loertscher L, Reed DA, Bannon MP, Mueller PS. Cardiopulmonary Resuscitation and Do-Not-Resuscitate Orders: A Guide for Clinicians. JAMA.2010; 123: 4-9. doi: 10.1016/j. amjmed.2009.05.029. 
24. Schober A, Sterz F, Herkner H., Locker GJ., Heinz G., Fuhrmann V., Sitzwohl C. et al. Post-resuscitation care at the emergency department with critical care facilities - a lengthof-stay analysis. Resuscitation. 2011;82: 853-58. doi: 10.1016/j. resuscitation.2011.03.004. Epub 2011 Mar 22.

25. Sladjana A. A prediction survival model for out-of-hospital cardiopulmonary resuscitations. Journal of Critical Care. 2011; 26: 223.e11-223.e18. doi: http://dx.doi.org/10.1016/j. jerc.2010.06.001

26. Petris A, Cimpoesu D, Costache I, Rotariu I. Decizia "de a nu resuscita" (I). Probleme de etică în cursul resuscitării cardiopulmonare. Revista Română de Bioetică. 2011; 9(2): 40 49. Romanian.

Publish in International Archives of Medicine

International Archives of Medicine is an open access journal publishing articles encompassing all aspects of medical science and clinical practice. IAM is considered a megajournal with independent sections on all areas of medicine. IAM is a really international journal with authors and board members from all around the world. The journal is widely indexed and classified Q1 in category Medicine. 